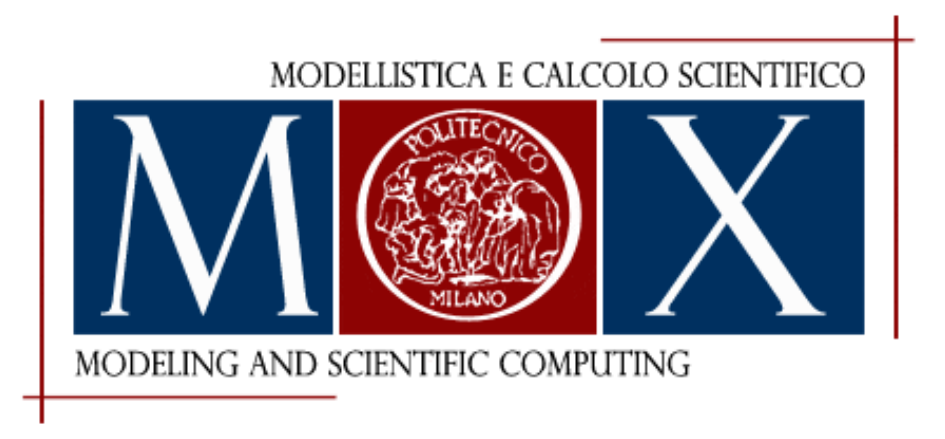

MOX-Report No. 39/2015

\title{
A semiparametric Bayesian joint model for multiple mixed-type outcomes: an Application to Acute Myocardial Infarction
}

Guglielmi, A.; Ieva, F.; Paganoni, A.M.; Quintana, F.A.

$$
\text { MOX, Dipartimento di Matematica }
$$

Politecnico di Milano, Via Bonardi 9 - 20133 Milano (Italy) 


\title{
A semiparametric Bayesian joint model for multiple mixed-type outcomes: an Application to Acute Myocardial Infarction
}

\author{
Alessandra Guglielmi $^{1}$, Francesca Ieva $^{2}$, Anna Maria Paganoni $^{1}$ \\ and FERnANDo A. QuintanA ${ }^{3}$ \\ ${ }^{1}$ Politecnico di Milano, Dipartimento di Matematica, Milano (ITALY) \\ ${ }^{2}$ Università degli Studi di Milano, Department of Mathematics, Milano (ITALY) \\ ${ }^{3}$ Pontificia Universidad Católica de Chile, Departamento de Estadística, Santiago (CHILE)
}

July 28,2015

AMS Classification: 62F15, 62P10, 62J12.

\begin{abstract}
We propose a Bayesian semiparametric regression model to represent mixed-type multiple outcomes concerning patients affected by Acute Myocardial Infarction. Our approach is motivated by data coming from the ST-Elevation Myocardial Infarction (STEMI) Archive, a multi-center observational prospective clinical study planned as part of the Strategic Program of Lombardy, Italy. We specifically consider a joint model for a variable measuring treatment time and in-hospital and 60-day survival indicators. One of our motivations is to understand how the various hospitals differ in terms of the variety of information collected as part of the study. We are particularly interested in using the available data to detect differences across hospitals. In order to do so
\end{abstract}


we postulate a semiparametric random effects model that incorporates dependence on a location indicator that is used to explicitly differentiate among hospitals in or outside the city of Milano. The model is based on the two parameter Poisson-Dirichlet prior, also known as the Pitman-Yor process prior. We discuss the resulting posterior inference, including sensitivity analysis, and a comparison with the particular submodel arising when a Dirichlet process prior is assumed.

Keywords: Bayesian clustering; Bayesian nonparametrics; two parameter Poisson-Dirichlet process prior; Random-effects models; Random Partition Models; Unbalanced binary outcomes.

\section{Introduction}

Studies with multiple outcomes that are used to properly characterize an effect of interest are becoming increasingly more common nowadays. In particular, in a clinical context, multiple outcomes are often used to characterize the patient's status or the performances of health care service with respect to patients' management (see, for example Normand, 2008; Parekh et al., 2011; AHRQ, 2015).

This paper is concerned with the analysis of data collected in a clinical registry named STEMI Archive (Lombardia, 2009; Ieva, 2013), which is a result of a wider comprehensive project, namely The Strategic Program "Exploitation, integration and study of current and future health databases in Lombardy for Acute Myocardial Infarction"; for additional information, visit http://ima.metid.polimi.it This project is funded by the Italian Ministry of Health. Its main goal is to enhance the integration of different sources of health information so as to automate and streamline clinicians' work flow, and that all the data collected can be generally used. We specifically consider outcomes of patients with ST segment elevation myocardial infarction (STEMI) diagnosis admitted to a hospital. STEMI is caused by an occlusion of a coronary artery which causes an ischemia that, if untreated, can damage heart cells and make them die (infarction). It is fundamental for the patient's recovery to do a reperfusion therapy (i.e. restoration of the blood flow to the ischemic tissue) as quickly as possible, since its benefits decrease highly non-linearly with treatment delay. All patients in the study were treated with Percutaneous Transluminal Coronary Angioplasty (PTCA). 
Data were recorded in a registry collecting clinical outcomes, process and time indicators measuring the way the health care structures manage the patients, and personal information on patients with STEMI diagnosis admitted to hospitals of Lombardy. These data were combined with information coming from the standard administrative database, so as to obtain out of hospital mortality (i.e., mortality for any reason). Data in the survey are grouped by hospital of admission. This automatically induces a policy issue about the effect that such grouping may have on patients' outcome, which is the main motivation for this work.

We propose a Bayesian nonparametric hierarchical model that includes a cluster analysis, aimed at identifying profiles or hospital behaviors that may affect the outcome at patient level. In particular, we introduce a multivariate regression model, where the response has three mixed-type components. The components are, respectively: (i) the door to balloon time (DB), i.e. the time between the admission to the hospital and the PTCA; (ii) the in-hospital survival; and (iii) the survival after 60 days from admission. The first response (continuous) is essential in quantifying the efficiency of health providers, since it plays a key role in the success of the therapy; the second is the basic treatment success indicator, while the third concerns a 60-days period, during which the treatment effectiveness, in terms of survival and quality of life, can be truly evaluated. Note that the last two responses are binary, so that, as a whole, the multivariate response is of mixed type.

The modeling of multiple outcomes from data collected in STEMI Archive was previously discussed in Ieva et al. (2013), under a semiparametric frequentist bivariate probit model. Their aim was analyzing the relationship among in-hospital mortality and a treatment effectiveness outcome in presence of endogeneity, a problem which poses serious limitations to covariate adjustment since the use of classical techniques may yield biased and inconsistent estimates. In contrast, we focus on a joint model for the grouped outcomes. In particular, as discussed below, our focus is on finding relevant groups of hospitals in terms of patient-specific characteristics, which may assist in further planning and policy making.

In recent years, there has been a considerable interest on developing models that overcome the challenges posed by the the mixed nature of outcomes. Sammel et al. (1997) discuss a model for mixed discrete and continuous outcomes where the multiple outcomes correlate through subject-specific latent variables. The observed outcomes are thus manifestations 
of unobserved latent variables, and conditionally on these, the outcome components are assumed independently distributed according to the exponential family, whose parameters are allowed to be a function of the latent variables as well as other component-specific covariates. Dunson and Herring (2005) proposed a Bayesian latent variable model for clustered mixed outcomes that allows nonlinear relationships between covariates and latent variables, and uses multiple latent variables for different types of outcome as well as covariate-dependent modifications of these relationships. In contrast, a single linear combination of the covariates is used to predict multiple outcomes simultaneously in the Bayesian multivariate model by Weiss et al. (2011), where correlations among outcomes are modeled by latent variables. Bello et al. (2012) present a hierarchical Bayesian extension of bivariate generalized linear models whereby functions of the variance-covariance matrices are specified as different linear combinations of fixed and random effects.

A somewhat different approach for bivariate outcomes of mixed type arises by factorizing the joint distribution of outcomes and introducing latent variables to model the correlation among the multiple outcomes. The main idea of this method is to write the likelihood as the product of the marginal distribution of one outcome and the conditional distribution of the second given the previous one. In particular Cox and Wermuth (1992) discuss two factorization models for a continuous and a binary outcome as functions of covariates. In Catalano and Ryan (1992) and Fitzmaurice and Laird (1995) the factorization approach is extended to clustered data.

Our approach is based on factorizations. In particular, we factorize the likelihood factor for the three responses for each patient as the product of (i) the marginal likelihood of the continuous response (DB time); (ii) the distribution of the in-hospital survival given DB time; and of (iii) the 60-days survival, given the previous two. All these conditional distributions lie within the class of univariate generalized linear mixed models, with random-effects given by hospital intercepts. Covariates corresponding to the other regression parameters include those related to hospital admission, patient's clinical status at hospital admission, and patient's general health status. A full description of available covariates is given in Section 2. To deal with differences across hospitals, we adopt a nonparametric random effects approach, with a random distribution function that is allowed to vary with an indicator 
that explicitly differentiates among hospitals in or outside the city of Milano. We adopt an ANOVA-Dependent Pitman-Yor process prior for hospital effects, that is, a family of distribution of dependent random probability measures with (marginal) almost surely discrete trajectories that generalize the Dirichlet process (DP). Such priors induce a random partition of the hospital labels. As we discuss later, the Pitman-Yor process (PY) process includes two parameters that allow for increased flexibility in the prior clustering structure compared to the DP. This is particularly useful to achieve one of our main goals, that is, to estimate a latent clustering among hospitals from the dataset, identifying groups of care providers affecting outcomes at patient level in a similar way. In this context, a cluster analysis of the hospitals is straightforward, based on posterior estimates of the induced random partition parameter itself. Besides marginal posterior inference on all relevant parameters, we discuss predictive inference for new hospitals, and hospitals clustering. Moreover, some competitor models are considered and compared to the proposed one through predictive goodness-of-fit tools.

The rest of this paper is organized as follows. Section 2 states the main inference questions that drive the analysis, also giving a complete description of the responses and available covariates. Section 3 describes the adopted model in detail, and posterior inference, implementation details and comparison among different models are discussed in Section 4. Final comments are given in Section 5 .

\section{Motivation and Data Description}

We consider a dataset coming from the integration of a clinical registry named STEMI Archive (Lombardia, 2009; Ieva, 2013), with data from the administrative health database. Our focus is on data from patients in any of the hospitals in Lombardy, and the analysis of their time to treatment, their in-hospital and 60-days survival outcomes. Our goals are (i) to understand the effect of other factors on the selected outcome variables; (ii) to compare marginal posterior distributions of the different nonparametric components; (iii) to compare hospital performances by means of a cluster analysis; and (iv) to make predictions for new hospitals entering the study (e.g., hospitals outside the region, but in districts gravitating towards Lombardy). 
A similar problem, with a related dataset, was already considered in Guglielmi et al. (2014). However, there are differences in the two statistical problems tackled, and in the two datasets analyzed. In particular, in Guglielmi et al. (2014), the interest was on building a model for predicting only the in-hospital survival after STEMI at patients' level, and to provide model-based clustering of the providers. This goal was achieved via a univariate regression model having patient's in-hospital survival as the response. On the other hand, it is known that one of the crucial factors influencing in-hospital survival for STEMI patients is treatment time (see for example De Luca et al., 2004; Antoniucci et al., 2002), which was considered as a fixed covariate in the latter paper. Here we focus not only on the causal relationship among in-hospital survival and treatment time, but we also aim at uncovering determinants (both logistic and environmental) affecting times to treatment, jointly with the two survival outcomes. What strongly motivates the different dataset we analyze here is the statistical interest in survival beyond discharge time, which would be of great help to better understand the disease progression and health recovery of STEMI patients.

The dataset at hand includes information about $n=697$ patients treated with PTCA in $J=33$ hospitals of Lombardy, 12 of these located in Milan. The number of patients per hospital ranges from a minimum of 5 to a maximum of 60 , with mean 21 . The available information about each patient are then the hospital of admission, the mode of admission (a binary variable indicating whether the patient was delivered by rescue units of 118, which is the Italian toll-free emergency number), demographic features such as age and gender, the severity of infarction, risk factors (such as diabetes, smoking and high cholesterol), times to treatment or intervention, and process indicators within the pre- and in-hospital phase. We resumed all the information content of the dataset through the following list:

- DB $\left(Y_{1}\right)$ : the time between the admission to the hospital (Door) and primary angioplasty (Balloon);

- ALIVEIN $\left(Y_{2}\right)$ : the in-hospital survival;

- ALIVE60 $\left(Y_{3}\right)$ : the survival after 60 days from admission.

These three variables represent the outcome. Observe that the dataset is strongly unbalanced: $96.84 \%$ of patients are alive after the discharge and $98.37 \%$ of them are alive after 
60 days. The sample mean and standard deviation of $D B$ in the log-scale are 4.452 and 0.551 .

The available covariates are listed here:

- ACCESS: 0 if the patient came to hospital by any rescue unit, 1 otherwise (by own means). The sample mean is 0.597 ;

- ECG: time of the first electrocardiogram (minutes). The sample mean is 9.671 (std. dev. 18.296);

- WE: 1 if the admission was on holiday, weekend or between $6 \mathrm{pm}-8 \mathrm{am}, 0$ otherwise. The sample mean is 0.469 ;

- AGE: age of the patient (years). The sample mean is 64.651 (std. dev. 13.122);

- GENDER: gender of the patient; 1 when male, 0 female. The sample mean is 0.776 ;

- RISK: 1 if patient had at least four among the following risk factors: diabetes, smoking, hypertension, cholesterol, vasculopathy, previous infarction, chronic kidney disease, 0 otherwise. The sample mean is 0.105 ;

- KILLIP: 1 if the infarction was severe (Killip class 3 or 4), 0 otherwise (Killip class 1 or 2). The sample mean is 0.060 ;

- EF: ejection fraction at admission to hospital, i.e. the volumetric fraction of blood pumped out of the ventricle with each heart beat (\%). The sample mean is 47.858 (std. dev. 9.663);

- COMP: 1 if there were complications after the primary angioplasty, 0 otherwise. The sample mean is 0.386 ;

- CKD: 1 if the patient had chronic kidney disease, 0 otherwise. The sample mean is 0.080

- STres: 1 if the treatment was not effective, 0 otherwise; this covariate is quantified by physicians as equal to 0 if there was a reduction of at least $70 \%$ in the ST-elevation within one hour later angioplasty. The sample mean is 0.198 ; 
- HOSPITAL: hospital of admission of the patient;

- MILAN: 1 if the hospital is located in Milano, 0 otherwise; the sample mean is 0.445 .

Treatment times (DB and ECG) are computed with respect to time of admission at the hospital.

In Figure 1 we report the boxplots of the DB time (in logarithmic scale) stratified by hospitals. The large variability and overdispersion due to the grouped nature of the data suggests that it is reasonable to assume a random effect on the grouping factor.

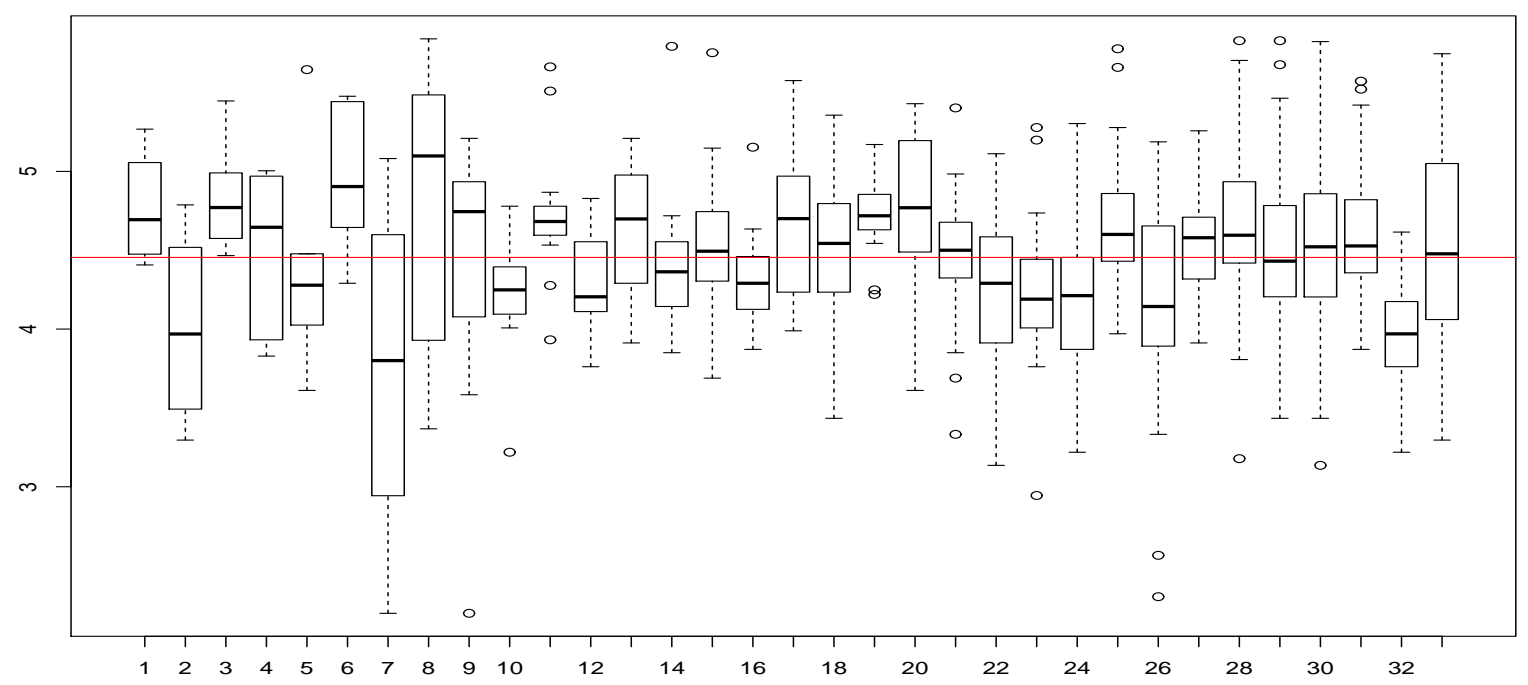

Figure 1: Boxplots of the DB time (in the log scale) stratified by hospitals. The red line is the global median of all DB times.

Figure 2 (left panel) shows the sample survival proportions (at discharge and after 60 days after discharge from hospital) per hospital. The right panel shows the difference between the two survivals. Observe that for most cases, the in-hospital and 60-days survivals are very similar. The two hospitals where this difference is the largest are 8 and 22 . 

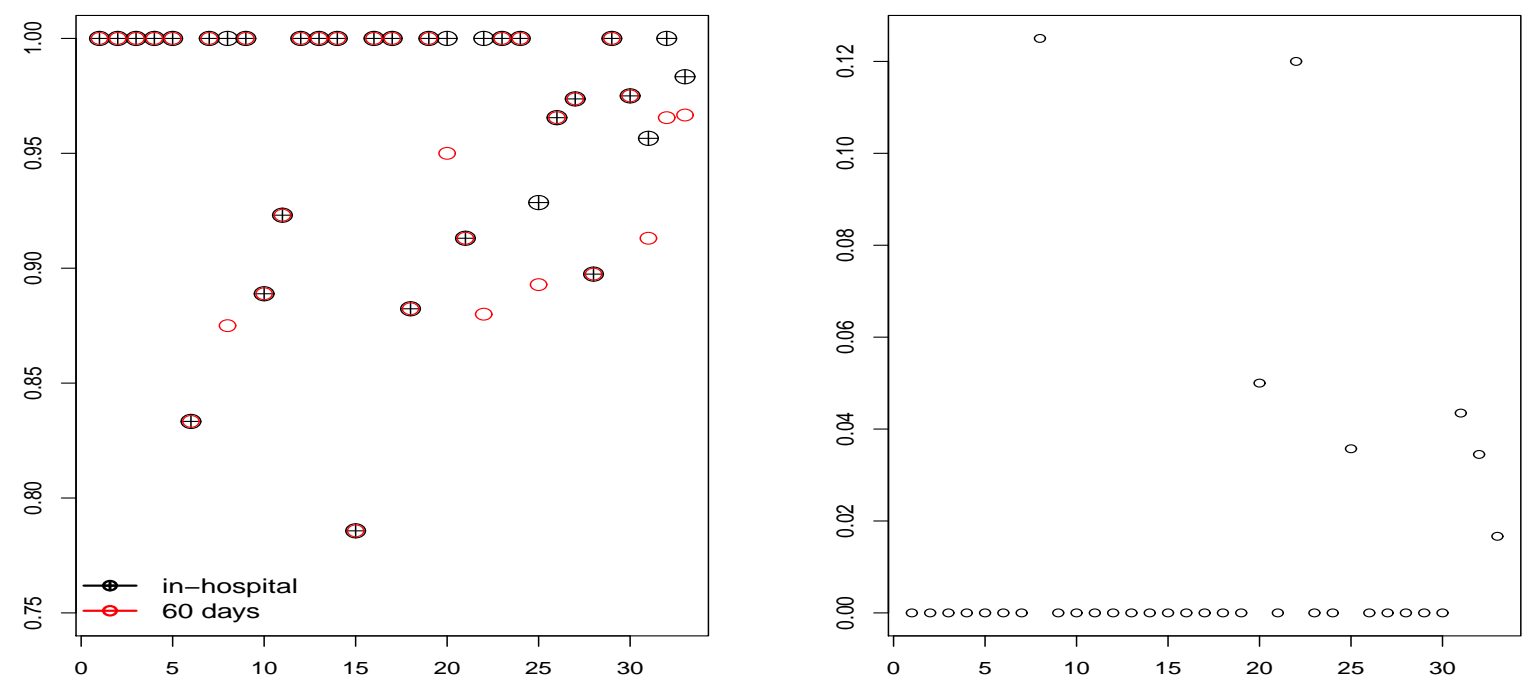

Figure 2: Sample survival proportions (at discharge and after 60 days) per hospital (left) and their difference (right).

\section{A multi-response Bayesian semiparametric model with Pitman-Yor process prior}

To achieve the goals described in Section 2, we propose a trivariate regression model of mixed types, according to the three outcome variables described before. We use a Bayesian semiparametric approach with a discrete random probability measure prior. This choice is also due to the flexibility they provide in modeling data, as well as the implied robustness against incorrect model specifications. See Müller and Quintana (2004) and Müller and Mitra (2013) for a thorough discussion on Bayesian Nonparametrics. Moreover, the discrete random measure model that we adopt as a prior for the hospital random-effects allows us to infer on a partition of the hospital labels. The prior we set here is the Pitman-Yor process (Pitman and Yor, 1997), which includes the (regular) DP chosen in Guglielmi et al. (2014) as a special case. Model details will be given below and in the next section.

In particular, we consider a generalized linear model for the response of patient $i$ treated in hospital $j, \mathbf{Y}_{j i}:=\left(Y_{j i 1}, Y_{j i 2}, Y_{j i 3}\right)=\left(\log \left(\mathrm{DB}_{j i}\right), \operatorname{ALIVEIN}_{j i}, \operatorname{ALIVE} 60_{j i}\right)$, with $i=1, \ldots, n_{j}$, 
$j=1, \ldots, J$. Since patients are admitted to hospitals, and one of the aims is to compare the hospitals themselves, it is natural and straightforward to consider generalized linear models with random intercepts to account for hospital variability. As usual, we assume that observations, given parameters and covariates, are independent. To facilitate model specification, we consider a conditional specification of the joint sampling model, conditional on parameters and covariates, as

$$
\mathcal{L}\left(Y_{j i 1} \mid \text { par, cov }\right) \times \mathcal{L}\left(Y_{j i 2} \mid Y_{j i 2}, \text { par, cov }\right) \times \mathcal{L}\left(Y_{j i 3} \mid Y_{j i 2}, Y_{j i 1}, \text { par, cov }\right)
$$

Our assumptions in this case are the following: $\mathcal{L}\left(Y_{j i 1} \mid\right.$ par, cov $)$ is a Gaussian linear regression, $\mathcal{L}\left(Y_{j i 2} \mid Y_{j i 1}\right.$, par, cov $)$ and $\mathcal{L}\left(Y_{j i 3} \mid Y_{j i 2}, Y_{j i 1}\right.$, par, cov $)$ are logistic regression models.

Before detailing the covariates at the three levels, we point out that we have made an extensive and thoughtful choice of covariates. First we consulted with our experts (cardiologists and health managers from different hospitals in Lombardy) to gain a better understanding of the covariates to include in a parametric regression model where $\mathbf{Y}_{j i}$ is the response, as in (1). The binary indicator MILAN of the geographical location of hospitals was included among the covariates to select, unlike the hospital label. Then, we adopted two priors for selecting the variables, i.e. the Normal Mixture of Inverse Gamma (NMIG) distributions and the SSVS spike-and-slab prior in Rockova et al. (2012); see notation and details of the priors there. In particular, under both priors, we fixed the variance of the spike and of the slab components equal to 0.001 and 10, respectively; here we report the selection considering all the covariates selected by the four highest posterior models under the NMIG prior. The other prior gives consistent results.

If $\boldsymbol{x}_{j i \ell}$ denotes the covariate vector for the sampling model at level $\ell=1,2,3$ of patient $i$ in hospital $j$, after covariates selection we got $\boldsymbol{x}_{j i 1}:=\left(\mathrm{ACCESS}_{j i}, \mathrm{ECG}_{j i}, \mathrm{WE}_{j i}, \mathrm{CKD}_{j i}\right)$, $\boldsymbol{x}_{j i 2}:=\left(\mathrm{EF}_{j i}, \mathrm{COMP}_{j i}, Y_{j i 1}, \mathrm{KILLIP}_{j i}\right)$, and $\boldsymbol{x}_{j i 3}:=\left(\mathrm{EF}_{j i}, \mathrm{GENDER}_{j i}, \mathrm{STres}_{j i}, \mathrm{KILLIP}_{j i}\right)$. Note that with a slight abuse of notation, we added $Y_{j i 1}$ to the set of covariates $\boldsymbol{x}_{j i 2}$.

It is interesting to note that, at first level, all the covariates $\left(\boldsymbol{x}_{j i 1}\right)$ related to logistic and organizational issues are retained: the way a patient is delivered to the emergency room, the time at which she/he receives the first ECG, and the time at which she/he arrives (recall this is coded as on/off hours), which are clearly related to the efficiency and the promptness of the treatment received. Finally, the presence/absence of chronic kidney disease (CKD) 
is also meaningful, because this condition may influence the time to intervention, as it is likely that more complex procedures will be required before undergoing surgery, since the radiocontrast agent may harm kidneys. On the other hand, in-hospital survival in STEMI patients undergoing angioplasty in this dataset will be modeled as depending on the initial patient's heart condition (EF), treatment time DB, severity of infarction (as indicated by KILLIP) and the presence of complications after PTCA (COMP). Finally, our model for mid-term survival (here ALIVE60) includes the initial heart condition (EF) and the severity of infarction (KILLIP) as well, and, of course, whether the treatment had been effective (STres); moreover, it results to be significantly influenced also by the gender of the patient (GENDER). Note that the age of the patient was never selected, even under different variances of the prior, or under the SSVS spike-and-slab prior. However, gender is usually highly correlated to age in STEMI patients (Trappolini et al., 2001; Vakili et al., 2001). Finally, it is worth mentioning that the selection included the binary indicator MILAN for explaining the first and the third responses, but we decided to include this information in the non-parametric component; see details below.

Now, once that the covariates have been selected, let us introduce the model. Recall that $i=1, \ldots, n_{j}$ indexes patients treated in hospital $j$, for $j=1, \ldots, J$. We assume that the conditional distributions in (1) are:

$$
\begin{gathered}
Y_{j i 1} \mid \mu_{j i}, \sigma_{j} \sim \mathcal{N}\left(\mu_{j i}, \sigma_{j}^{2}\right), \quad \mu_{j i}=\boldsymbol{\beta}_{1}{ }^{T} \boldsymbol{x}_{j i 1}+b_{\phi_{j} j}^{1} \\
Y_{j i 2} \mid p_{j i}, Y_{j i 1} \sim B e\left(p_{j i}\right), \quad \operatorname{logit}\left(p_{j i}\right)=\boldsymbol{\beta}_{2}{ }^{T} \boldsymbol{x}_{j i 2}+b_{\phi_{j} j}^{2} \\
Y_{j i 3} \mid q_{j i}, Y_{j i 1}, Y_{j i 2} \sim\left\{\begin{array}{ll}
B e\left(q_{j i}\right) & \text { if } Y_{j i 2}=1 \\
\delta_{0} & \text { if } Y_{j i 2}=0
\end{array}, \quad \operatorname{logit}\left(q_{j i}\right)=\boldsymbol{\beta}_{3}{ }^{T} \boldsymbol{x}_{j i 3}+b_{\phi_{j} j}^{3} .\right.
\end{gathered}
$$

Here, as usual, $\delta_{0}$ denotes the degenerate distribution in 0 .

Observe that in (2)-(4), parameters $b_{\phi_{j} j}^{1}, b_{\phi_{j} j}^{2}, b_{\phi_{j} j}^{3}, \sigma_{j}$ represent hospital-specific random effects; the former three are random intercepts, while the latter is the standard deviation of the first response. Notation $\phi_{j}$ is a dummy variable indicating if the hospital is in Milano, or outside the city. In fact, the management of emergencies is pretty different in or outside the city, due to the different concentration of providers on the territory and to the related 
accessibility. These are expected to affect times to intervention and, through them, patients' outcomes. On the other hand, Milano can be considered as a hub, resulting more attractive to patients and then characterized by a wider spectrum of cases. Therefore, it seems reasonable to establish an explicit difference in the random effects, according to whether the hospital is in $\left(b_{1}^{1}\right)$ or outside of $\left(b_{0}^{1}\right)$ Milano. In fact, a feature of our model is that we allow the entire shape of the random effects distributions to change according to this geographical characteristic. This is exactly the reason why we consider a dependent nonparametric prior specification; see details below.

Consequently, our inference will mainly focus on parameter

$$
\boldsymbol{\theta}=\left(\boldsymbol{\beta}_{1}, \boldsymbol{\beta}_{2}, \boldsymbol{\beta}_{3},\left(b_{0 j}^{1}, b_{1 j}^{1}, b_{0 j}^{2}, b_{1 j}^{2}, b_{0 j}^{3}, b_{1 j}^{3}, \sigma_{j}, j=1, \ldots, J\right)\right)
$$

where $J$ is the number of hospitals in the dataset. We assume a priori independence of all components of $\boldsymbol{\theta}$ and:

$$
\boldsymbol{\beta}_{1} \sim \mathcal{N}_{4}\left(\mathbf{0}, 100 \mathbb{I}_{4}\right), \quad \boldsymbol{\beta}_{2} \sim \mathcal{N}_{4}\left(\mathbf{0}, 100 \mathbb{I}_{4}\right), \quad \boldsymbol{\beta}_{3} \sim \mathcal{N}_{4}\left(\mathbf{0}, 100 \mathbb{I}_{4}\right)
$$

and for $j=1 \ldots, J$,

$$
\begin{aligned}
\sigma_{j} & \stackrel{\text { iid }}{\sim} U(0,10), \\
\left(b_{0 j}^{1}, b_{1 j}^{1}, b_{0 j}^{2}, b_{1 j}^{2}, b_{0 j}^{3}, b_{1 j}^{3}\right) \mid P & \stackrel{i i d}{\sim} P, \quad P \sim P Y\left(a, g, P_{0}\right) .
\end{aligned}
$$

By $P \sim P Y\left(a, g, P_{0}\right)$ we mean that $P$ is a Pitman-Yor process (Pitman and Yor, 1997), sometimes known as the two-parameter Poisson-Dirichlet process, with parameters $0 \leq a<1$ and $g>-a$, while $P_{0}$ is a probability measure on $\mathbb{R}^{6}$. When $a=0$, the DP case is recovered. Observe that the nonparametric part specification (7) together with the sampling model (2) - (4) results in a generalization of the ANOVA-DDP prior in De Iorio et al. (2004).

For ease of computation it is useful to introduce the stick-breaking representation for $P$ (Pitman, 1995):

$$
P=\sum_{i=1}^{\infty} V_{i} \delta_{\boldsymbol{\tau}_{i}}, \quad \text { where }\left\{V_{i}\right\} \perp\left\{\boldsymbol{\tau}_{i}\right\},
$$

and $\boldsymbol{\tau}_{i}$ s are iid according to some distribution and $\left\{V_{i}\right\}$ are stick-breaking weights, i.e.

$$
V_{1}=Z_{1}, \quad V_{j}=Z_{j} \prod_{i=1}^{j-1}\left(1-Z_{i}\right) \quad j \geq 2, \quad Z_{i} \stackrel{i n d}{\sim} \operatorname{Beta}(1-a, g+i a), \quad i=1,2, \ldots
$$


It is well-know that a random sample from a distribution $F$ that is assigned a discrete random prior probability measure such as (7) induces a random partition $\rho$ of corresponding labels. In this case, the partition is induced on the hospitals labels $\{1,2, \ldots, J\}$ by (7). Thus, hospitals would be grouped by identifying those with identical random intercept, according to (2)-(4). In this way, we will be allowed to carry out model-based clustering by computing a summary estimate of the posterior distribution of the random partition $\rho$. The induced partition structure is more general than that coming from the particular DP case.

Next, we assume for the locations $\boldsymbol{\tau}_{i} \in \mathbb{R}^{3}$ the parameterization usually adopted for the ANOVA-DDP prior, i.e.

$$
\tau_{i}=\tau_{0 i}+\tau_{1 i} \eta_{i}
$$

where $\eta_{i}$ is 1 if the patient $i$ was admitted to an hospital in Milano, and 0 otherwise. On the whole, the location parameters are identified by $\left(\tau_{01 i}, \tau_{11 i}, \tau_{02 i}, \tau_{12 i}, \tau_{03 i}, \tau_{13 i}\right)$, and we assume they are iid from the base probability measure $P_{0}$ on $\mathbb{R}^{6}$ given by the product measure of six independent Gaussian distributions with random means and variances:

$$
\begin{gathered}
P_{0}=\mathcal{N}\left(m_{1}, \lambda_{1}^{2}\right) \times \mathcal{N}\left(m_{2}, \lambda_{2}^{2}\right) \times \mathcal{N}\left(m_{3}, \lambda_{3}^{2}\right) \times \mathcal{N}\left(m_{4}, \lambda_{4}^{2}\right) \times \mathcal{N}\left(m_{5}, \lambda_{5}^{2}\right) \times \mathcal{N}\left(m_{6}, \lambda_{6}^{2}\right) \\
\left(m_{1}, \ldots, m_{6}, \lambda_{1}, \ldots, \lambda_{6}\right) \sim \pi_{m} \times \pi_{\lambda}
\end{gathered}
$$

The prior clustering is controlled by hyperparameters $a$ and $g$ in (7). For fixed $a$, the number of clusters is stochastically increasing with $g$. This can be seen as a "rich gets richer" property of the PY that is also shared by the DP. This effect is attenuated by the parameter $a$, which can be thought of as a discount parameter.

The model specification is completed by assuming

$$
m_{i} \stackrel{\text { ind }}{\sim} \mathcal{N}\left(m_{i 0}, \sigma_{i 0}^{2}\right), \quad \lambda_{i} \stackrel{i i d}{\sim} \mathcal{U}\left(0, \lambda_{0}\right) \quad i=1, \ldots, 6
$$

but other weakly informative choices could be considered as well.

Before discussing specific results, we point out that we tried extensive posterior simulation experiments under several types of priors for the PY parameters $a$ and $b$. These experiments found a number of posterior simulation problems, such as poor mixing of certain parameters, but also, none of the models we tried produced a better fitting to the data than what we will describe in Section 4. 


\section{Posterior inference}

\subsection{Model details}

It is quite common in practice to consider a truncated version of the stick-breaking representation (8)-(9) so as to work with a finite mixture model (see Ishwaran and Zarepour, 2002). This is achieved by considering a number of components, say $H$, and setting $Z_{H}=1$ in (9). Posterior inference can then be implemented through a standard Gibbs sampler algorithm, which we coded in JAGS (Plummer, 2003) with the aid of R ( R Development Core Team, 2012). In what follows, we always use the first 50,000 iterations as burn-in, and saving every 75-th iteration after that, to complete a Monte Carlo posterior sample of size 5,000. Standard convergence diagnostics criteria such as those available in the $\mathrm{R}$ package CODA (Plummer et al., 2006) were applied to all parameters, indicating that convergence had been achieved.

To fit the model, we selected hyperparameter values that reflect lack of prior information, in other words, a vague yet proper prior distribution. Specifically, we chose

$$
m_{i 0}=0, \quad \sigma_{i 0}^{2}=25, \quad i=1, \ldots, 6, \quad \lambda_{0}=5 .
$$

The prior for the Pitman-Yor process was specified as follows. We fixed $a=0.3$ and $b=0.5$ so that the prior mean and variance of $K_{J}$ are equal to 5.285 and 6.113 , respectively.

\subsection{Posterior Summaries}

Table 1 reports the 95\% posterior credible intervals, as well as the posterior marginal probability on the negative reals, for the fixed-effects parameters at all the three levels. From the reported inference, it is clear that patients who were not delivered by the 118 service and/or arrived at weekends or nights are penalized in terms of DB time. Furthermore, as expected, an increase of ECG time yields an increase of DB, testifying the importance of executing promptly ECG to the patients when infarction diagnoses are suspected. Also the presence of CKD results significant: as we said before, this makes sense since complications may arise when treating a patient whose kidneys do not work properly.

For the in-hospital survival probability (level 2), patients with a more severe infarction 


\begin{tabular}{|l|ccc|l|ccc|c|ccc|}
\hline \multicolumn{5}{|c}{ Level 1 } & \multicolumn{5}{|c|}{ Level 2} & \multicolumn{4}{c|}{ Level 3 } \\
\hline Parameter & $2.5 \%$ & $97.5 \%$ & $p^{-}$ & Parameter & $2.5 \%$ & $97.5 \%$ & $p^{-}$ & Parameter & $2.5 \%$ & $97.5 \%$ & $p^{-}$ \\
\hline \hline ACCESS & -0.015 & 0.133 & 0.059 & EF & 0.692 & 1.732 & 0 & EF & 0.708 & 1.578 & 0 \\
\hline ECG & 0.106 & 0.161 & 0 & COMP & -5.543 & -1.229 & 1 & GENDER & -1.120 & 0.851 & 0.604 \\
\hline WE & 0.012 & 0.141 & 0.013 & $Y_{1}$ & -1.288 & 0.588 & 0.764 & STres & -1.520 & 0.284 & 0.912 \\
\hline CKD & 0.032 & 0.318 & 0.007 & KILLIP & -2.382 & 0.151 & 0.963 & KILLIP & -2.491 & -0.301 & 0.994 \\
\hline
\end{tabular}

Table 1: Posterior 95\% credibility intervals for the fixed-effects parameters; $p^{-}$is the posterior probability that the parameter is negative.

and/or with complications after PTCA are penalized. Also an elevated DB time $\left(Y_{1}\right)$ decreases significantly the survival probability. On the other hand, the ejection fraction at admission (EF) has a positive effect on in-hospital survival.

Similarly, EF and KILLIP have positive and negative effect, respectively, on 60-days survival, while the negative results of PTCA, quantified by the STres, plays a negative role as expected. Even if it is clear that gender has a negative effect, this is rather moderate.

In Figures 3 and 4 we provide posterior 95\% CIs of the hospital random intercepts; in all these figures, as before, the hospitals are ordered from left to right by increasing number of patients available in the sample. The last two intervals in each panel represent predictions for random intercepts corresponding to two hypothetical new hospitals, located in (continuous red) and outside (dashed blue) Milano. It is clear that there is a hospital effect in the first DB times (see the variability of the estimates in Figure 3). In particular, hospitals located in Milano show a lower variability than those located outside. On the other hand, there is much more homogeneity in the random intercepts at the second and third level. This behavior can be explained because all the coronary units treat patients according to general standards, which yields rather uniform hospital performances in terms of in-hospital survival. 


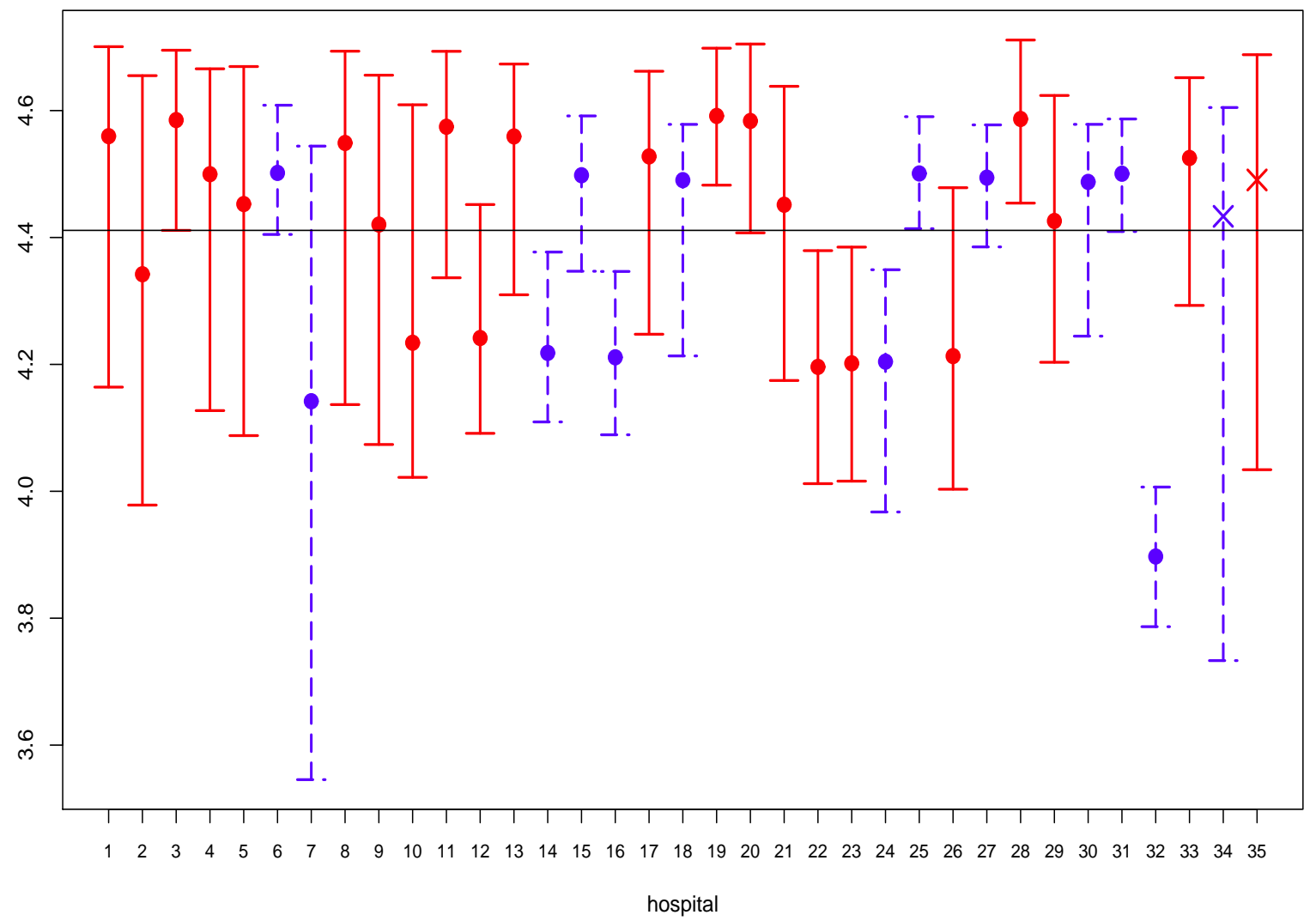

Figure 3: Posterior CI of $b_{j}^{1}$, the random effect parameters at the first level of the likelihood. Hospitals located in Milano are depicted in dashed (blue) lines, those outside Milano in solid (red) lines, and bullets are the posterior medians. The last two intervals represent predictions (medians are marked by crosses) for random intercepts corresponding to two hypothetical new hospitals, located in (red) and outside (blue) Milano. For reference, the horizontal line represent the mean of all displayed means.

We have also computed posterior predictive estimates of the different nonparametric component of the mixing measure. Figure 5 displays posterior estimates of the components of $P=\sum_{i=1}^{H} V_{i} \delta_{\boldsymbol{\tau}_{i}}$. In particular, the first row shows the first (left) and second (right) predicted components (level one in the likelihood, i.e. the posterior of $\sum_{1}^{H} V_{i} \delta_{\tau_{01 i}}$ and of $\sum_{1}^{H} V_{i} \delta_{\tau_{01 i}+\tau_{11 i}}$ ), and similarly for the third and fourth (level two in the likelihood) and fifth and sixth (third level in the likelihood) components. Dashed (blue) lines correspond to 

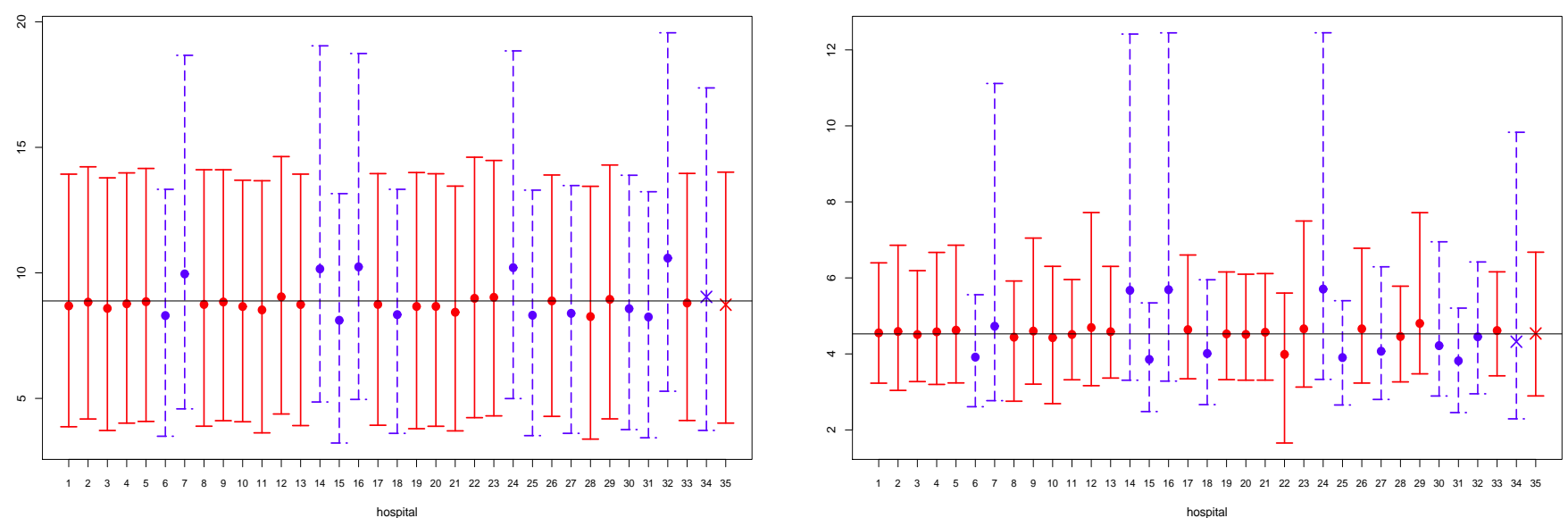

Figure 4: Posterior 95\% CIs of the random intercepts $b^{2}$ (left) and $b^{3}$ (right): hospitals located in Milano are depicted in dashed (blue) lines, those outside Milano in solid (red) lines, and bullets are the posterior medians. The last two intervals represent new random intercepts for a hospital in and outside Milano, with crosses representing posterior medians.

0.025 and 0.925 quantiles, while the solid central lines represent the respective means (i.e. the Bayesian estimates). The last 50 iterations are superimposed (in red). The picture shows a difference in the variability of the sampled trajectories, at least at the second and third level. The results in Figure 5 are in agreement with the information conveyed by the marginal posterior distributions of $m_{2}, m_{4}$ and $m_{6}$ (not reported here), which represent the average difference in the random effect parameters between hospitals in or out of Milano. In fact, the marginal posterior distribution of $m_{2}$ is concentrated around 0 (posterior mean and variance are -0.152 and 0.152 , respectively), denoting that on average there is no Milano effect on the $\log D B$ response. The marginal posterior distributions of $m_{4}$ and $m_{6}$ are much more spread out.

\subsection{Posterior inference on clustering}

As pointed out earlier, the discrete trajectories of the nonparametric prior assumption imply a clustering of the hospitals. We found that the posterior mean and variance of $K_{J}$, the number of groups among hospitals, are 5.602 and 3.795, respectively, with a posterior mode 
of 4 (but 5 has a posterior probability very close to that of 4). Figure 6 displays the whole estimated posterior distribution.

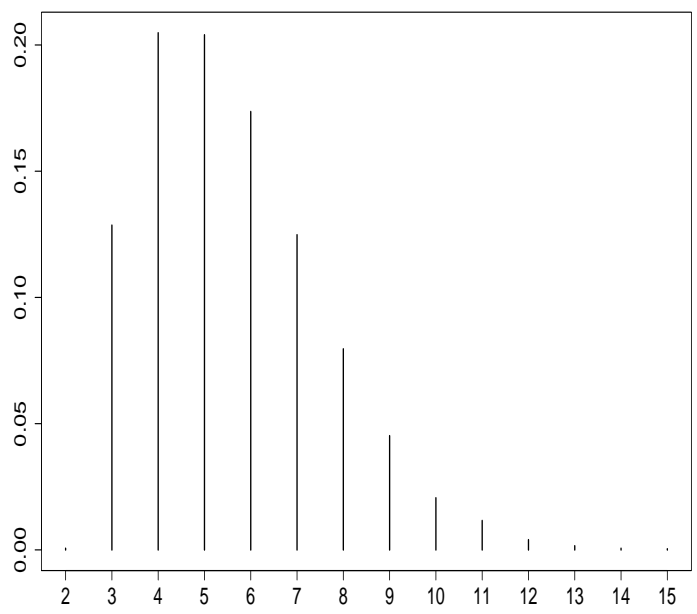

Figure 6: Posterior distribution of the number of groups among the hospitals.

The Bayesian cluster estimate was here computed as the random partition of the hospital labels $\{1,2, \ldots, 33\}$ that minimizes the posterior expectation of Binder's loss function, as proposed in Lau and Green (2007); this function assigns cost $w$ when two elements are wrongly clustered together and cost $u$ when two elements are erroneously assigned to different clusters. For equal misclassification $\operatorname{costs} w$ and $u$, we obtained 9 clusters in total, but only 4 with sizes larger that 1 . Table 2 reports the four non-singleton groups in the cluster estimate. We underline that this estimate agrees with the least squares estimate of Dahl (2006).

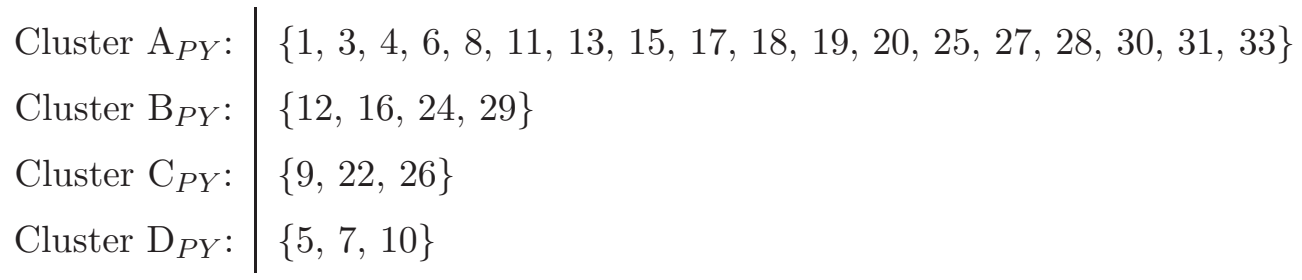

Table 2: Hospital clusters with sizes larger than 1 from the proposed model under the Pitman-Yor process prior when $a=0.3$ and $b=0.5$.

We have computed sample means of responses and covariates per hospital clusters in Table 2, 
averaging over all patients of all hospitals in each group. Table 3 reports those values. Cluster $A_{P Y}$ (the most populated) could be characterized as grouping patients with the highest DB times and the lowest survival rates. This suggests that the hospitals in this group may have procedures that could be improved to achieve a better performance. Note also that the KILLIP rate in cluster $A_{P Y}$ is the highest among the groups, and higher than the sample grand mean (0.06). In contrast, clusters $B_{P Y}$ and $D_{P Y}$ group hospitals with patients having less severe infarction. In addition, clusters $B_{P Y}$ and $D_{P Y}$ differ in the associated complications exhibited by patients and in the way patients accessed the hospitals. Finally, cluster $C_{P Y}$ contains only hospitals outside Milano and the corresponding patients have on average the lowest ECG values in the sample.

\begin{tabular}{|c|cccc|}
\hline groups & $A_{P Y}$ & $B_{P Y}$ & $C_{P Y}$ & $D_{P Y}$ \\
\hline No. hospitals & 18 & 4 & 3 & 3 \\
\hline No. patients & 398 & 106 & 72 & 29 \\
\hline$Y_{1}$ (DB) & 115.455 & 87.953 & 83.514 & 80.966 \\
\hline$Y_{2}$ (ALIVEIN) & 0.955 & 1.000 & 0.986 & 0.966 \\
\hline$Y_{3}$ (ALIVE60) & 0.940 & 1.000 & 0.944 & 0.966 \\
\hline MILANO & 0.475 & 0.387 & 0.000 & 0.276 \\
\hline ACCESS & 0.555 & 0.642 & 0.597 & 0.414 \\
\hline ECG & 10.487 & 10.708 & 6.764 & 8.448 \\
\hline WE & 0.440 & 0.481 & 0.417 & 0.448 \\
\hline CKD & 0.090 & 0.028 & 0.111 & 0.103 \\
\hline EF & 47.621 & 49.519 & 49.208 & 47.310 \\
\hline COMP & 0.465 & 0.236 & 0.333 & 0.586 \\
\hline KILLIP & 0.083 & 0.000 & 0.056 & 0.000 \\
\hline AGE & 64.500 & 65.104 & 64.931 & 68.276 \\
\hline GENDER & 0.802 & 0.708 & 0.819 & 0.724 \\
\hline STres & 0.176 & 0.226 & 0.250 & 0.069 \\
\hline
\end{tabular}

Table 3: Responses and covariates summaries by clusters in Table 2, for $a=0.3, b=0.5$. 


\subsection{Predictive goodness-of-fit}

We consider now predictive checks for the proposed model. We first computed the log pseudo-marginal likelihood (LPML) statistic (Geisser and Eddy, 1979) for this model. This corresponds to the product, in the $\log$ scale, of the conditional predictive density of the responses, i.e.

$$
L P M L=\sum_{i=1}^{n} \log \left(C P O_{i}\right),
$$

where $C P O_{i}$ represents the conditional density (evaluated at $\boldsymbol{y}_{j i}$ ), of $\boldsymbol{Y}_{j i}$, given all the other observations. We also computed the mean squared error $M S E$ of the prediction errors, i.e. the mean of $S E_{i}$ over hospitals given by

$$
S E_{i}=\left(Y_{j i 1}-\hat{\mu}_{j i}\right)^{2}+\left(Y_{j i 2}-\hat{p}_{j i}\right)^{2}+\left(Y_{j i 3}-\hat{q}_{j i}\right)^{2}
$$

where the hat denotes the posterior expectation of the corresponding parameters. Following Gelman et al. (2014), we also considered the Watanabe-Akaike information criterion (WAIC), computed as the log pointwise predictive density, incorporating bias corrections. Specifically, we computed

$$
W A I C_{1}=l p p d-p_{W A I C_{1}} \quad \text { and } \quad W A I C_{2}=l p p d-p_{W A I C_{2}},
$$

where $l p p d$ is the log pointwise predictive density, i.e. the product (in the log scale) of the conditional densities (evaluated at $\boldsymbol{y}_{j i}$ ), of $\boldsymbol{Y}_{j i}$, given all the data, and then adding the two alternative corrections $p_{W A I C_{1}}$ and $p_{W A I C_{2}}$ for effective number of parameters to adjust for overfitting. The bias correction $p_{W A I C_{1}}$ is similar to the bias correction in the definition of the DIC, while $p_{W A I C}$ is the sum of the posterior variances of the conditional density of the data. For further details, see Section 3 of Gelman et al. (2014). The computed predictive goodness-of-fit measures are in Table 4.

\subsection{Comparison with competitor models}

When introducying the proposed model (2)-(7), we aimed at justifying all the choices we made. However it is natural to wonder whether simpler models could give similar inference. While we are pretty satisfied about the conditional distribution of data, given parameters 


\begin{tabular}{|l||c|c|c|}
\hline random effects prior & $P Y(a=0.3, b=0.5)$ & $P Y(a=0, b=1.53)$ & Parametric \\
\hline \hline LPML & -594.40 & -593.037 & -596.053 \\
\hline MSE & 0.282 & 0.282 & 0.280 \\
\hline$W A I C_{1}$ & -581.847 & -581.082 & -582.096 \\
\hline$W A I C_{2}$ & -589.613 & -588.974 & -591.105 \\
\hline
\end{tabular}

Table 4: Predictive goodness-of-fit measures when the prior of the random effects is the Pitman-Yor process with parameters $a=0.3, b=0.5$, or $a=0, b=0.5$ (i.e. the Dirichlet process with parameter $b=1.53)$, or parametric.

(see (2)-(4)), we acknowledge that other simpler priors could be considered here. We examine some alternatives next.

First, let us consider the same prior as before, but now setting $a=0$, which reduces nonparametric prior to a Dirichlet process (DP). In this case we fixed $b=1.53$ to match the prior mean under the Pitman-Yor process prior component. In particular we now have $\mathbb{E}\left(K_{j}\right)=5.302$ and $\operatorname{Var}\left(K_{J}\right)=3.130$. The estimated fixed effects under the DP prior are very similar to those in Table 1 (data not shown).

We have also computed the posterior predictive estimates of the different nonparametric components in the mixing measure. Comparing Figures 5 and 7 we find that the means are almost identical, but the quantile curves in the DP case are a bit more separated from the mean, suggesting a slightly increased posterior uncertainty in the corresponding posterior distributions.

The cluster estimate we obtained in this case contains 8 groups, but only 5 have sizes larger that 1. Table 5 describes the largest groups in the cluster estimate. Again, this results agrees with what is obtained when using the least squares method of Dahl (2006). The partitions are now a bit different, with the big cluster containing less points than the PY case.

Table 6 reports sample means of responses and covariates per hospital clusters, averaging over the entire set of patients in all hospitals forming each group in Table 5. The largest cluster here is similar to that in Table 3, but with two less members. However the other 


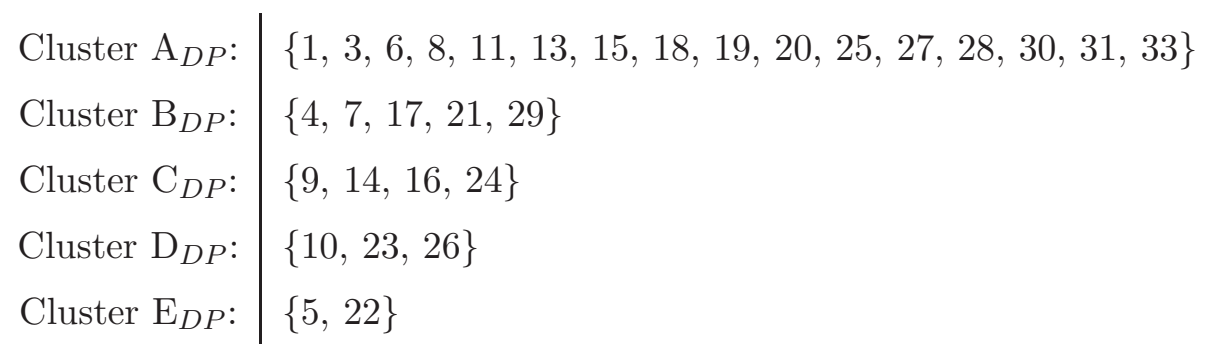

Table 5: Hospital clusters with size larger than 1 from the proposed model under the PitmanYor process prior when $a=0$ and $b=1.53$; this corresponds to assuming a Dirichlet process prior with parameter $b=1.53$.

groups cannot be as clearly interpreted in terms of responses as in the case of the proposed PY process model. For instance, the mean values of $D B$ are ordered in Table 3, unlike in Table 6. Under the previous case, the hospitals with highest averages of both in-hospital and 60-days survival were in the second largest cluster. In contrast, here the best hospitals in terms of in-hospital survival are split in the third and fifth largest clusters, while those with highest average 60-days survival are in the largest third and second groups.

As a final remark on comparison between these competitors, we should underline that, according to Table 4, the DP prior is slightly superior. The differences in values between the several criteria reported there are minimal though, and we still prefer the Pitman-Yor model because of a clearer interpretation of the selected partition, as discussed earlier.

We also considered a non-dependent version of the proposed Pitman-Yor model, which eliminates the in/out of Milano indicator in the random effects. Specifically, we change $b_{\phi_{j} j}^{\ell}$ to $b_{j}^{\ell}$ for $\ell=1,2,3$ and $j=1, \ldots, J$ in $(2)-(4)$. Doing so we obtained predictive check values comparable to those already reported in Table 4 , but miss the subtle yet relevant differences in the predictive curves (left versus right panels) in Figures 5 or 7.

Another natural comparison involves a model with a parametric prior for random effects. Under this alternative model, the likelihood is the same as in (2) - (4), but now the random effects are assumed to be i.i.d. draws from the baseline distribution (10). Fitting this model produced fixed effects estimates quite similar to those in Table 1. Regarding random effects, the CIs for the $b_{1}$ parameters are similar to those in Figure 3, but differences arise for the $b_{2}$ and $b_{3}$ terms (data not shown). The predictive check measures for this model 


\begin{tabular}{|c|ccccc|}
\hline groups & $\mathrm{A}_{D P}$ & $\mathrm{~B}_{D P}$ & $\mathrm{C}_{D P}$ & $\mathrm{D}_{D P}$ & $\mathrm{E}_{D P}$ \\
\hline No. hospitals & 16 & 5 & 4 & 3 & 2 \\
\hline No. patients & 375 & 99 & 73 & 72 & 37 \\
\hline DB & 115.648 & 101.374 & 86.521 & 74.625 & 85.351 \\
\hline ALIVEIN & 0.952 & 0.980 & 1.000 & 0.958 & 1.000 \\
\hline ALIVE60 & 0.936 & 0.980 & 1.000 & 0.958 & 0.919 \\
\hline MILANO & 0.504 & 0.081 & 0.753 & 0.000 & 0.000 \\
\hline ACCESS & 0.544 & 0.697 & 0.534 & 0.583 & 0.595 \\
\hline ECG & 9.899 & 13.818 & 12.356 & 5.556 & 5.108 \\
\hline WE & 0.445 & 0.434 & 0.466 & 0.514 & 0.486 \\
\hline CKD & 0.093 & 0.091 & 0.027 & 0.056 & 0.189 \\
\hline EF & 47.211 & 49.869 & 51.068 & 47.181 & 48.946 \\
\hline COMP & 0.469 & 0.313 & 0.329 & 0.417 & 0.108 \\
\hline KILLIP & 0.083 & 0.061 & 0.014 & 0.056 & 0.000 \\
\hline AGE & 64.485 & 66.293 & 64.178 & 63.181 & 66.541 \\
\hline GENDER & 0.795 & 0.687 & 0.753 & 0.819 & 0.865 \\
\hline STres & 0.176 & 0.232 & 0.288 & 0.208 & 0.270 \\
\hline
\end{tabular}

Table 6: Responses and covariates summaries by clusters in Table 5 , when $a=0, b=1.53$; this corresponds to a Dirichlet process prior for the random effect parameters.

are also presented in Table 4. Note that, except for the mean squared error (MSE), the non-parametric alternatives produce better fits to the data.

\section{Conclusions}

We have presented a framework for semiparametric Bayesian modeling of mixed-type multiple outcomes for Acute Myocardial Infarction patients admitted to hospitals in Lombardy; we considered patients with STEMI diagnosis and treated with PTCA. Specifically, we have proposed a Bayesian nonparametric hierarchical model for cluster analysis, aimed at identifying hospital behaviour that may affect the outcome at patient level. We have considered 
a conditional specification of the joint model for three responses: the door to balloon time (DB), the in-hospital survival and the survival after 60 days from admission. Each conditional is a generalized linear model with random intercepts to account for hospital variability. We postulated a nonparametric prior for the random effects that incorporates dependence on a location indicator, which is used to explicitly differentiate among hospitals in or outside the city of Milano. The random effects are a sample from the Pitman-Yor process, more flexible than Dirichlet process prior, however encompassing the DP. We have provided Bayesian estimates of the random effect parameters, predictive inference for the nonparametric components of the prior, as well as cluster estimates for the grouping of the hospitals. Finally, we have considered a number of competitor models, either purely parametric, or with a nonparametric component. Though all the models provide similar results in terms of the fixed effects estimates, the most flexible model (where the random effects are modeled from the Pitman-Yor process) seems the one able to explain better the underlying clustering structure. Code of the proposed model is available from authors upon request.

\section{Acknowledgments}

This work is within the strategic programme Exploitation, integration and study of current and future health databases in Lombardia for Acute Myocardial Infarction. The authors thank Regione Lombardia Healthcare Division for having funded and sustained the project, Lombardia Informatica SpA for having provided the data and all the physicians who contributed to the STEMI archive planning and data collection.

The first author wishes to thank people at Departamento de Estadística at PUC, Chile, for their kind hospitality. The fourth author was partially funded by grant FONDECYT 1141057.

\section{References}

AHRQ (2015). Agency for Healthcare Research and Quality.

URL http://www . ahrq.gov/professionals/prevention-chronic-care/decision/mcc 
Antoniucci, D., Valenti, A., Migliorini, A., and et al (2002). "Relation of Time to Treatment and Mortality in patients with Acute Myocardial Infarction undergoing Primary Angioplasty." The American Journal of Cardiology, 89, 1248-1252.

Bello, N. M., Steibel, J. P., and Tempelman, R. J. (2012). "Hierarchical Bayesian modeling of heterogeneous cluster-and subject-level associations between continuous and binary outcomes in dairy production." Biometrical Journal, 54, 230-248.

Catalano, P. and Ryan, L. (1992). "Bivariate latent variable models for clustered discrete and continuous outcomes." Journal of the American Statistical Association, 87, 651-658.

Cox, D. and Wermuth, N. (1992). "Response models for binary and quantitative variables." Biometrika, 79, 441-461.

Dahl, D. B. (2006). "Model-based clustering for expression data via a Dirichlet process mixture model." In Do, K.-H., Müller, P., and Vannucci, M. (eds.), Bayesian inference for gene expression and proteomics, 201-218. Cambridge: Cambridge University Press.

De Iorio, M., Müller, P., Rosner, G., and MacEachern, S. (2004). "An ANOVA Model for Dependent Random Measures." Journal of the American Statistical Association, 99, $205-215$.

De Luca, G., Suryapranata, H., Ottervanger, J. P., and Antman, E. M. (2004). "Time delay to treatment and mortality in primary angioplasty for acute myocardial infarction: every minute of delay counts." Circulation, 109, 1223-1225.

Dunson, D. B. and Herring, A. H. (2005). "Bayesian latent variable models for mixed discrete outcomes." Biostatistics, 6, 11-25.

Fitzmaurice, G. and Laird, N. (1995). "Regression models for a bivariate discrete and continuous outcome with clustering." Journal of the American Statistical Association, 90, $845-852$.

Geisser, S. and Eddy, W. F. (1979). "A predictive approach to model selection." Journal of the American Statistical Association, 74, 153-160. 
Gelman, A., Hwang, J., and Vehtari, A. (2014). "Understanding predictive information criteria for Bayesian models." Statistics and Computing, 24, 997-1016.

Guglielmi, A., Ieva, F., Paganoni, A., Ruggeri, F., and Soriano, J. (2014). "Semiparametric Bayesian modeling for the classification of patients with high observed survival probabilities." Journal of the Royal Statistical Society, C, 63, 25-46.

Ieva, F. (2013). "Designing and mining a multicenter observational clinical registry concerning patients with Acute Coronary Syndromes." In Grieco, N., Marzegalli, M., and Paganoni, A. M. (eds.), New diagnostic, therapeutic and organizational strategies for patients with Acute Coronary Syndromes, 47-60. Springer.

Ieva, F., Marra, G., Paganoni, A. M., and Radice, R. (2013). "A semiparametric bivariate probit model for joint modeling of outcomes in STEMI patients." MOX reports 39, Politecnico di Milano.

URL http://mox.polimi.it/it/progetti/pubblicazioni/quaderni/39-2013.pdf

Ishwaran, H. and Zarepour, M. (2002). "Exact and approximate sum representations for the Dirichlet process." Canadian Journal of Statistics, 30, 269-283.

Lau, J. W. and Green, P. J. (2007). "Bayesian model based clustering procedures." Journal of Computational and Graphical Statistics, 16, 526-558.

Lombardia (2009). "Determinazioni in merito alla Rete per il trattamento dei pazienti con Infarto Miocardico con tratto ST elevato (STEMI)."

Müller, P. and Mitra, R. (2013). "Bayesian nonparametric inference-why and how." Bayesian analysis, 8, 269-302.

Müller, P. and Quintana, F. A. (2004). "Nonparametric Bayesian data analysis." Statistical science, 19, 95-110.

Normand, S.-L. T. (2008). "Some old and some new statistical tools for outcomes research." Circulation, 118, 872-884. 
Parekh, A., Goodman, R., Gordon, C., and Koh, H. (2011). "Managing Multiple Chronic Conditions: A Strategic Framework for Improving Health Outcomes and Quality of Life." Public Health Reports, 126, 460-471.

Pitman, J. (1995). "Exchangeable and partially exchangeable random partitions." Probability Theory and Related Fields, 102, 145-158.

Pitman, J. and Yor, M. (1997). "The two-parameter Poisson-Dirichlet distribution derived from a stable subordinator." The Annals of Probability, 25, 855-900.

Plummer, M. (2003). "JAGS: A program for analysis of Bayesian graphical models using Gibbs sampling."

Plummer, M., Best, N., Cowles, K., and Vines, K. (2006). "CODA: Convergence Diagnosis and Output Analysis for MCMC." R News, 6, 7-11.

R Development Core Team (2012). R: A Language and Environment for Statistical Computing. R Foundation for Statistical Computing, Vienna, Austria. ISBN 3-900051-07-0. URL http://www.R-project.org/

Rockova, V., Lesaffre, E., Luime, J., and Löwenberg, B. (2012). "Hierarchical Bayesian formulations for selecting variables in regression models." Statistics in medicine, 31, 12211237.

Sammel, M., Ryan, L. M., and Legler, J. M. (1997). "Latent Variable Models for Mixed Discrete and Continuous Outcomes." Journal of the Royal Statistical Society. Series B (Methodological), 59, 667-678.

Trappolini, M., Chillotti, F., Rinaldi, R., Trappolini, F., Coclite, D., Napoletano, A., and Matteoli, S. (2001). "Sex differences in incidence of mortality after acute myocardial infarction." Italian Heart Journal Suppl., 3, 759-766.

Vakili, B., Kaplan, R., and Brown, D. (2001). "Sex-Based Differences in Early Mortality of Patients Undergoing Primary Angioplasty for First Acute Myocardial Infarction." Circulation, 104, 3034-3038. 
Weiss, R., Jia, J., and Suchard, M. A. (2011). "A Bayesian model for the common effects of multiple predictors on mixed outcomes." Interface Focus, 1, 886-894. 

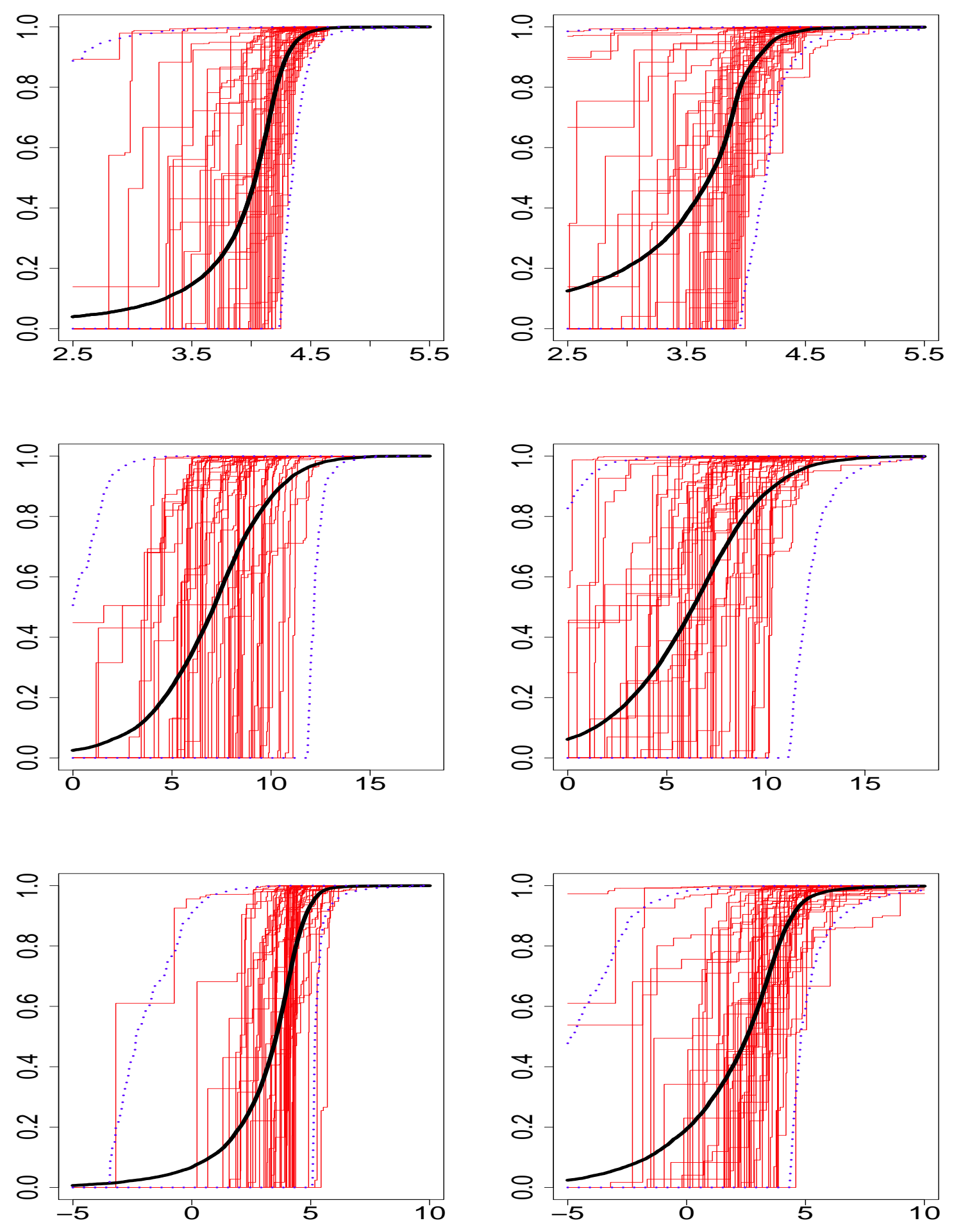

Figure 5: Posterior predictive 95\% CIs of the components of $P$. See the text for an explanation. Dashed (blue) lines correspond to quantiles, the solid central is the mean (the Bayesian estimates). The last 50 iterations are superimpgsed (in red). The left (right) column displays trajectories of the three random components related to hospitals outside (in) Milano. 

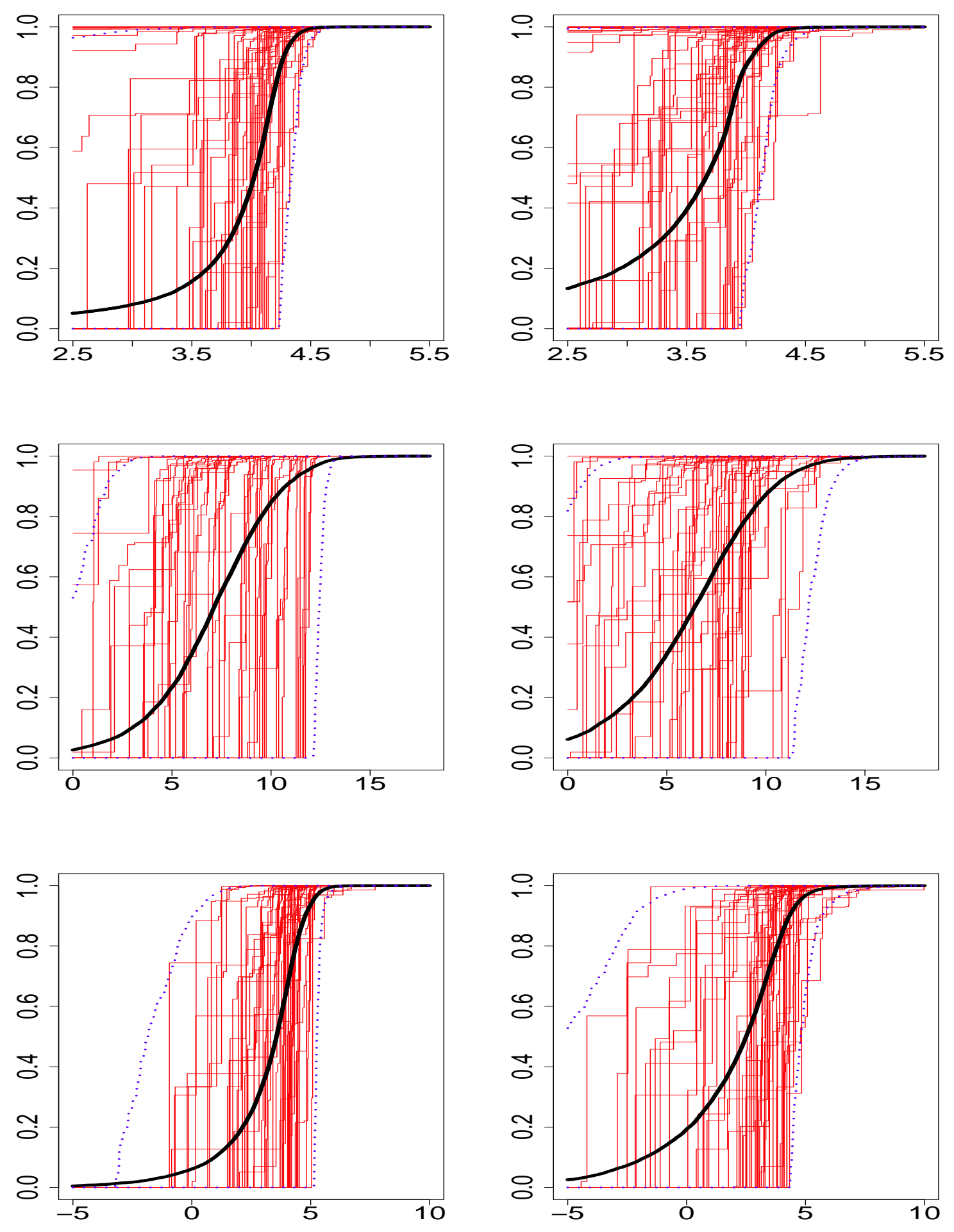

Figure 7: Posterior predictive 95\% CIs of the components of $P$ when $a=0, b=1.53$. Dashed (blue) lines correspond to quantiles, the solid central is the mean (the Bayesian estimates). The last 50 iterations are superimposed (in refor. The left (right) column displays trajectories of the three random components related to hospitals outside (in) Milano. 


\title{
MOX Technical Reports, last issues
}

\author{
Dipartimento di Matematica \\ Politecnico di Milano, Via Bonardi 9 - 20133 Milano (Italy)
}

38/2015 Grasso, M.; Menafoglio, A.; Colosimo, B.M.; Secchi, P.

Using Curve Registration Information for Profile Monitoring

36/2015 Fedele, M.; Faggiano, E.; Barbarotta, L.; Cremonesi, F.; Formaggia, L.; Perotto, S. Semi-Automatic Three-Dimensional Vessel Segmentation Using a Connected Component Localization of the Region-Scalable Fitting Energy

37/2015 Aletti, M.; Perotto, S.; Veneziani, A.

Educated bases for the HiMod reduction of advection-diffusion-reaction problems with general boundary conditions

35/2015 Manzoni, A.; Pagani, S.

A certified reduced basis method for PDE-constrained parametric optimization problems by an adjoint-based approach

31/2015 Pini, A.; Vantini, S.; Colombo, D.; Colosimo, B. M.; Previtali, B.

Domain-selective functional ANOVA for process analysis via signal data: the remote monitoring in laser welding

32/2015 Agasisti,T.; Ieva, F.; Masci, C.;Paganoni, A.M.

Does class matter more than school? Evidence from a multilevel statistical analysis on Italian junior secondary school students

33/2015 Fumagalli, A; Pasquale, L; Zonca, S.; Micheletti, S.

An upscaling procedure for fractured reservoirs with non-matching grids

34/2015 Bernardi, M.S.; Mazza, G.; Ramsay, J.O.; Sangalli, L.M.

A separable model for spatial functional data with application to the analysis of the production of waste in Venice province

28/2015 Taffetani, M.; Ciarletta, P.

Beading instability in soft cylindrical gels with capillary energy: weakly non-linear analysis and numerical simulations

30/2015 Pini, A.; Vantini, S.

Interval-wise testing for functional data 